\title{
O impacto do isolamento social na pandemia de COVID-19 no acesso ao tratamento e aos serviços de prevenção do HIV
}

\author{
The impact of social isolation on the COVID-19 pandemic on access to HIV treatment and prevention
}

services

El impacto del aislamiento social en la pandemia del COVID-19 en el acceso a los servicios de tratamiento y prevención del VIH

Recebido: 05/01/2021 | Revisado: 06/01/2021 | Aceito: 12/01/2021 | Publicado: 13/01/2021

\author{
Juliana da Silva Parente \\ ORCID: https://orcid.org/0000-0003-1019-8025 \\ Universidade Federal Fluminense, Brasil \\ E-mail:julianaparente@id.uff.br \\ Suely Lopes de Azevedo \\ ORCID: https://orcid.org/0000-0003-1107-3427 \\ Universidade Federal Fluminense, Brasil \\ E-mail:suelyazevedo@id.uff.br \\ Letícia da Fonseca Anacleto Moreira \\ ORCID: https://orcid.org/0000-0002-6288-1953 \\ Universidade Federal Fluminense, Brasil \\ Email:leticiaanacleto@id.uff.br \\ Larissa Murta Abreu \\ ORCID: https://orcid.org/0000-0002-7119-4370 \\ Universidade Federal Fluminense, Brasil \\ E-mail:larissamurtaabreu9@gmail.com \\ Lorrany Viana de Souza \\ ORCID: https://orcid.org/0000-0002-9251-4601 \\ Universidade Federal Fluminense, Brasil \\ E-mail:lorranyvianades@gmail.com
}

\begin{abstract}
Resumo
A Organização Mundial da Saúde, em março de 2020, declarou o COVID-19 como uma pandemia, recomendando medidas de prevenção, controle e tratamento da doença. Uma das principais medidas, o isolamento social, resultou na suspensão de diversos procedimentos nos serviços de saúde. A reorganização dos serviços voltados para o atendimento ambulatorial de patologias ocasionou perdas e consequências incalculáveis na continuidade do tratamento. O estudo tem como objetivo discutir as consequências das medidas de distanciamento social e a organização dos serviços ambulatoriais voltados para o atendimento das pessoas que vivem com HIV durante a pandemia de COVID-19. Método: revisão integrativa, realizada no Portal de Periódicos da CAPES, utilizando estudos disponíveis na íntegra, indexado no portal da PUBMED, contendo os descritores: HIV, Antiretroviral Therapy, Social Isolation. A amostra, resultou de 9 estudos selecionados após os critérios de inclusão e exclusão. Resultados: A pandemia interferiu diretamente no tratamento e na oferta de serviços para as pessoas que vivem com HIV. Evidenciou-se uma diminuição na rotina de testagem, reagendamentos ou supressão dos horários de atendimentos de rotina e diminuição da distribuição de medicamentos. Conclusão: Apesar da situação de risco, o atendimento nos serviços de saúde a populações-chave deve ser garantido, quali e quantitativamente, sendo responsabilidade dos gestores reorganizar os serviços, em busca de erradicar interrupções dos atendimentos e melhorar a assistência prestada.
\end{abstract}

Palavras-chave: HIV; COVID-19; Terapia antirretroviral; Isolamento social.

\begin{abstract}
The World Health Organization, in March 2020, declared COVID-19 as a pandemic, recommending measures for the prevention, control and treatment of the disease. One of the main measures, social isolation, resulted in the suspension of several procedures in health services. The reorganization of services aimed at the outpatient care of pathologies caused incalculable losses and consequences in the continuity of treatment. The study aims to discuss the consequences of social distance measures and the organization of outpatient services aimed at caring for people living with HIV during the COVID-19 pandemic. Method: an integrative review, carried out on the CAPES Journals Portal, using studies available in full, indexed on the PUBMED portal, containing the descriptors: HIV, Antiretroviral Therapy, Social Isolation. The sample resulted from 9 studies selected after the inclusion and exclusion criteria.
\end{abstract}


Results: The pandemic directly interfered in the treatment and provision of services for people living with HIV. There was a decrease in the routine of testing, rescheduling or suppression of the hours of routine appointments and a decrease in the distribution of medications. Conclusion: Despite the risk situation, care in health services for key populations must be guaranteed, qualitatively and quantitatively, and it is the managers' responsibility to reorganize the services, seeking to eradicate interruptions in care and improve the care provided.

Keywords: HIV; COVID-19; Antiretroviral therapy; Social isolation.

\section{Resumen}

La Organización Mundial de la Salud, en marzo de 2020, declaró al COVID-19 como una pandemia, recomendando medidas para la prevención, control y tratamiento de la enfermedad. Una de las principales medidas, el aislamiento social, resultó en la suspensión de varios procedimientos en los servicios de salud. La reorganización de los servicios orientados a la atención ambulatoria de patologías provocó pérdidas y consecuencias incalculables en la continuidad del tratamiento. El estudio tiene como objetivo discutir las consecuencias de las medidas de distancia social y la organización de servicios ambulatorios destinados a atender a las personas que viven con el VIH durante la pandemia de COVID-19. Método: revisión integradora, realizada en el Portal de Revistas CAPES, utilizando estudios disponibles en su totalidad, indexados en el portal PUBMED, que contienen los descriptores: VIH, Terapia Antirretroviral, Aislamiento Social. La muestra resultó de 9 estudios seleccionados después de los criterios de inclusión y exclusión. Resultados: La pandemia interfirió directamente con el tratamiento y la prestación de servicios para las personas que viven con el VIH. Hubo una disminución en la rutina de pruebas, reprogramación o supresión de las horas de citas de rutina y una disminución en la distribución de medicamentos. Conclusión: A pesar de la situación de riesgo, la atención en los servicios de salud para poblaciones clave debe estar garantizada, cualitativa y cuantitativamente, y es responsabilidad de los gestores reorganizar los servicios, buscando erradicar las interrupciones en la atención y mejorar la atención brindada.

Palabras clave: VIH; COVID-19; Terapia antirretroviral; Aislamiento social.

\section{Introdução}

A pandemia de Coronavírus (nomeada tecnicamente de COVID-19) é uma doença infecciosa recém-descoberta, causada por um novo Coronavírus, com aparência de coroa de espinhos, da família de vírus que causam infecções respiratórias, que se dissemina, principalmente, por gotículas de saliva ou secreção nasal quando uma pessoa infectada tosse ou espirra, sendo desconhecido até o momento vacinas ou tratamentos específicos para sua cura (Brasil, 2020a).

A COVID-19 é altamente perigosa devido as suas complicações respiratórias o que o dificulta seu controle, já acometeu o mundo com altas taxas de mortalidade, principalmente, entre a população de idosos e aqueles com problemas de saúde crônicos pré-existentes, como doenças cardiovasculares, diabetes, doenças respiratórias, pessoas vivendo com HIV, câncer e demais doenças autoimunes, pois esses indivíduos quando infectados, têm maior probabilidade de desenvolver a forma grave da doença (Moura \& Costa, 2020).

Os primeiros casos da doença, causada pelo novo Coronavírus, foram registrados em Wuhan, na China, sendo o vírus descoberto em 31 de dezembro de 2019. Devido ao seu poder de disseminação, a doença se difundiu rapidamente por todo o mundo, o que fez com que a Organização mundial de saúde (OMS), em 11 de março de 2020, definisse a doença COVID-19 como uma pandemia com significativos impactos na economia, nos sistemas de saúde e na população (OPAS, 2020).

De acordo com a OPAS e OMS (2020), onde é atualizado periodicamente a Vigilância Global para a infecção humana por Coronavírus - COVID-19, os dados recentes apontam para o fato de que até o dia 17 de dezembro de 2020 foram confirmados no mundo 72.851 .747 casos de COVID-19 (642.738 novos em relação ao dia anterior) e 1.643 .339 mortes (12.407 novas em relação ao dia anterior). Segundo Brasil (2020b) até o dia 21 de dezembro de 2020 foram confirmados 7.263.619 casos de COVID-19 2.187.291 óbitos confirmados. Sendo assim, o perfil epidemiológico da doença demonstra como ela é perigosa, exigindo do Ministério da saúde implementar medidas emergenciais, seguras e resolutivas para que a doença possa ser controlada e suas consequências minimizadas. 
A revisão se justifica pela necessidade de estudos nacionais que visem a atenção aos cuidados envolvendo prevenção, diagnóstico de HIV, servindo como ameaça ao tratamento e distribuição de medicamentos. Nesta perspectiva, este artigo tem o objetivo de refletir acerca do impacto da COVID-19 no serviço de saúde e a influência da pandemia e distanciamento social nesse contexto.

O isolamento social é uma das medidas de prevenção durante as pandemias, que tem o objetivo de reduzir a transmissão de novos vírus, mas que pode trazer graves repercussões. Os atendimentos nos serviços de saúde foram diretamente influenciados e precisaram se reorganizar, levando em conta seu potencial de bloqueio da transmissão viral e de minimizar os riscos da comunidade. É justamente nesses momentos de crise que a sociedade brasileira percebe a importância do sistema único de saúde (SUS) que garante o direito universal à saúde. Neste contexto, a pandemia atingiu as populações vulneráveis, o que gerou incertezas sobre quais seriam as melhores estratégias a serem utilizadas para seu enfrentamento. Os desafios foram ainda maiores, pois pouco se sabia sobre as características de transmissão da COVID-19, num contexto de grande desigualdade social, com populações vivendo em condições precárias, sem saneamento e em situação de aglomeração.

HIV é a sigla em inglês referente ao vírus da imunodeficiência humana que se dissemina através dos fluidos corporais, afetando células específicas do sistema imune, sendo essas, as células $\mathrm{CD} 4$, ou células $\mathrm{T}$, podendo levar à síndrome da imunodeficiência adquirida (AIDS). Uma vez contraído, a pessoa viverá com o vírus para sempre. Por enquanto ainda não há cura, mas o HIV pode ser controlado com a terapia antirretroviral aumentando a expectativa e qualidade de vida e diminuindo a porcentagem de transmissão. A progressão é bem documentada, se não tratada se torna fatal, destruindo o sistema imunológico, resultando na AIDS. Há três estágios da doença: Infecção aguda, fase assintomática e AIDS. O tratamento ajuda em todos os estágios e pode desacelerar e prevenir a progressão (UNAIDS, 2020a).

Há muitas pessoas HIV positivas que vivem anos sem apresentar sintomas e sem desenvolver a doença. Porém podem transmitir o vírus pelas relações sexuais desprotegidas, compartilhamento de seringas contaminadas e por transmissão vertical. Sendo a prevenção a barreira mais importante nesta fase. A baixa imunidade culmina no aparecimento de doenças oportunas que se aproveitam da fraqueza do organismo, quando o estágio mais avançado é atingido (AIDS) muitas vezes por não saber da infecção ou não seguir o tratamento indicado, pode vir a apresentar hepatites virais, tuberculose, pneumonia, toxoplasmose e alguns tipos de câncer (Brasil, 2020c).

Os medicamentos antirretrovirais (ARVS) agem inibindo a multiplicação do HIV, evitando o enfraquecimento do sistema imunológico, tornando a doença uma condição crônica controlável. Por isso, o uso regular dos ARVS é fundamental para garantir o controle da doença e prevenir a evolução e transmissão. A adesão à terapia antirretroviral (TARV) traz múltiplos benefícios, como o aumento da disposição, da energia e do apetite, ampliação da expectativa e qualidade de vida e o não desenvolvimento de doenças oportunistas. Desde 1996, o Brasil distribui gratuitamente pelo SUS (Sistema Único de Saúde) todos os medicamentos antirretrovirais e, desde 2013, o SUS garante tratamento para todas as pessoas vivendo com HIV (Brasil, 2020d).

A prevenção combinada constitui em uma estratégia que faz uso simultâneo de abordagens de prevenção (biomédica, comportamental e socioestrutural), são essas: a testagem regular para o HIV, que pode ser realizada gratuitamente no Sistema Único de Saúde (SUS); a prevenção da transmissão vertical; o tratamento das infecções sexualmente transmissíveis e das hepatites virais; a imunização para as hepatites A e B; programas de redução de danos para usuários de álcool e outras substâncias; profilaxia pré-exposição (PrEP); profilaxia pós-exposição (PEP); e o tratamento de pessoas que já vivem com HIV (Brasil, 2020e). 
Dias et al (2020) reflete que estar em isolamento social pela COVID-19 envolve muitas questões que as pessoas jamais imaginariam vivenciar, principalmente aquelas que tinham uma vida agitada e que pouco ficavam dentro de suas casas, seja pela hiperatividade, fuga da realidade ou a necessidade de trabalhar, estudar, divertir-se, visitar familiares, amigos etc.

O medo e ansiedade de contrair o vírus são grandes implicações e barreiras para as pessoas que precisam sair de suas casas para buscar assistência à saúde. Além de encontrar dificuldades ao tentar marcar consultas, tratamentos, exames complementares e/ou diagnósticos, consequência da redução do número de profissionais de saúde no atendimento na rede básica, principalmente nos programas de saúde, e a reorganização dos serviços, a fim de evitar aglomerações e reduzir a transmissão do vírus.

Isso remete à reflexão de que ao término dessa pandemia, poderá existir uma demanda reprimida de pessoas necessitando de assistência à saúde, por terem retardado a realização dos seus exames anuais de rotina ou por estarem apresentando complicações decorrentes de doenças crônicas prévias ou adquiridas, durante o período em que se mantiveram em isolamento social, visto que, em razão do medo de contraírem o vírus, não buscaram atendimento ou simplesmente não conseguiram agendamento.

O momento atual vivenciado por toda sociedade, impõe reflexão, transformações e ressignificações. Neste sentido, o artigo busca elucidar o impacto do novo Coronavírus na prestação do serviço de saúde as pessoas vivendo com HIV no contexto mundial, e a partir da revisão identificar contribuições de manejo e ações de cuidados no tratamento e prevenção de HIV no cenário do isolamento social e crise política, sanitária e social. Fazendo conexão dos trabalhos disponíveis dos serviços mundiais, devido a lacunas e escassez de pesquisas brasileiras, sendo preciso fazer uma busca com maior abrangência.

\section{Método}

Estudo de revisão integrativa, a partir de uma pesquisa bibliográfica, descritiva e qualitativa que busca, através de um determinado conhecimento atual sobre uma temática específica, conduzir de modo a identificar, analisar e sintetizar resultados de estudos independentes que abordam o mesmo assunto, podendo contribuir em uma repercussão favorável na qualidade dos cuidados prestados ao paciente.

Segundo Souza et al (2010) o impacto da utilização da revisão integrativa não se dá somente pelo desenvolvimento de políticas, protocolos e procedimentos, mas também no pensamento crítico que a prática diária necessita, é a mais ampla abordagem metodológica referente às revisões, permitindo a inclusão de variados estudos para uma compreensão completa do fenômeno. Incorpora um vasto leque de propósitos: definição de conceitos; revisão de teorias e evidências; e análise de problemas metodológicos. As revisões da literatura, normalmente devem ser extensas, aprofundadas e detalhadas evitando trabalhos superficiais e tendenciosos (Pereira et al., 2018).

A busca por estudos relacionados à temática ocorreu em 9 de novembro de 2020, identificando evidências científicas no Portal de Periódicos da CAPES, utilizando o critério de seleção para os periódicos disponíveis, indexação ao PUBMED. Utilizaram-se os descritores classificados referidos nos Descritores em Ciências da Saúde (DeCS), utilizando o operador booleano "AND": (HIV) AND (COVID-19) AND (Antiretroviral Therapy) AND (Social Isolation).

Para dar início às buscas partimos da questão norteadora que foi desenvolvida e sustentada a partir da estratégia PICo, que exige elementos que a fundamentam, sendo P- População, I- Interesse, Co- Contexto. Considerando estes elementos, definiu-se a seguinte estrutura: P- Pessoas vivendo, I- Acesso ao tratamento e prevenção do HIV, Co- Isolamento social na pandemia de COVID-19 (Santos, Pimenta \& Nobre, 2007). Logo chegando a formulação da pergunta: Qual o impacto do isolamento social durante a pandemia do COVID-19 na adesão de condutas de prevenção e de tratamento de HIV? 
Os critérios de inclusão após a leitura do título e resumo para seleção dos estudos científicos foram os artigos que abordam a temática e respondem a questão norteadora, disponíveis online na íntegra e que apresentavam os descritores publicados nos idiomas inglês, espanhol e português. Os critérios de exclusão após a leitura do título e resumo foram artigos que não abordavam a temática e não respondiam a questão norteadora. No recorte temporal de Janeiro de 2020 a dezembro de 2020.

Na Figura 1, é apresentado o caminho metodológico percorrido para a escolha dos artigos por meio dos descritores associados e o quantitativo de produções bibliográficas analisadas.

Figura 1. Fluxograma adaptado do modelo PRISMA 2009 utilizado na seleção dos estudos.

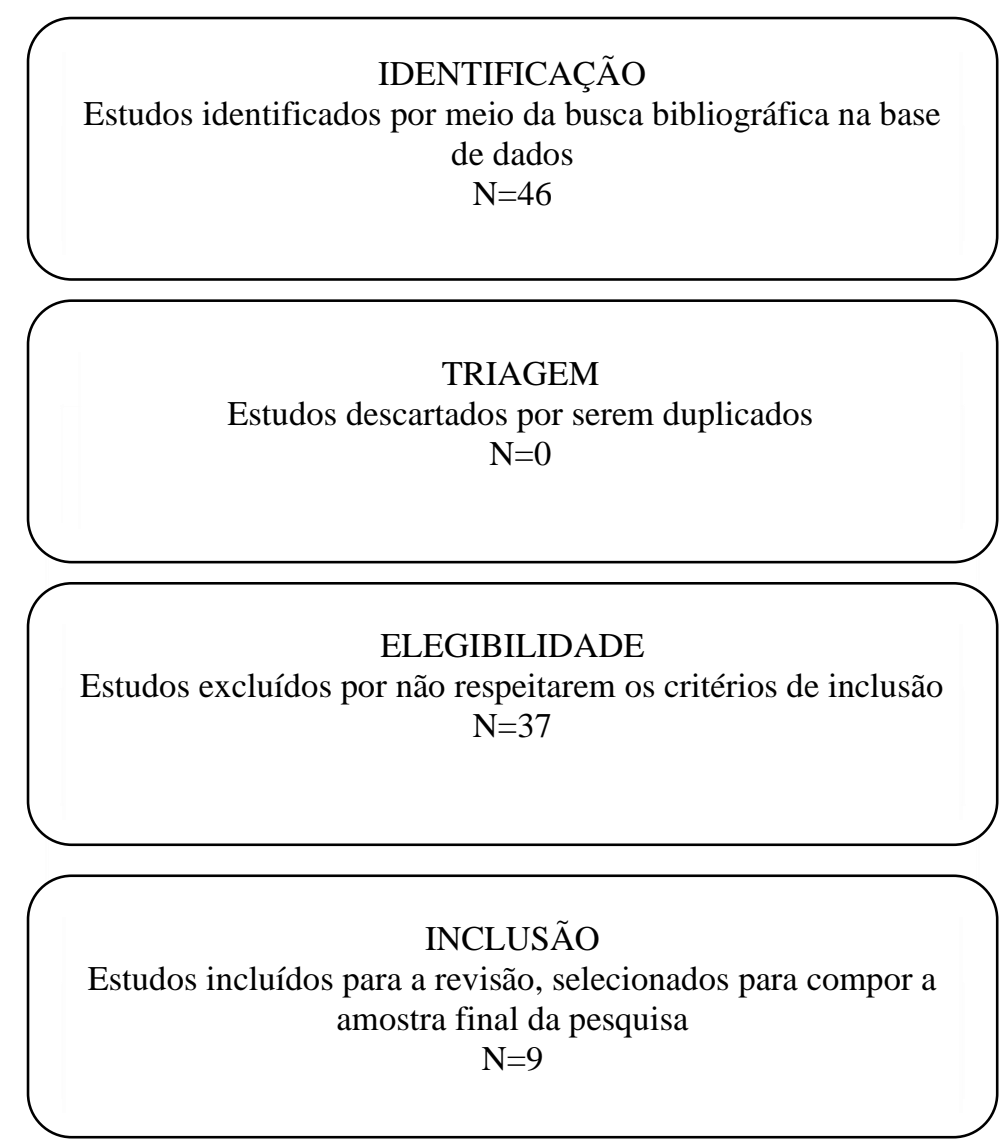

Fonte: Autores, adaptado do modelo PRISMA (2009).

Após leitura dos estudos, aplicação dos critérios de inclusão e exclusão foram selecionados 9 artigos, foi realizado uma leitura crítica dos selecionados para caracterizá-los de acordo com as metodologias utilizadas para compor a amostra final.

\section{Resultados}

Evidenciou-se que as ações de cuidados no tratamento e prevenção da HIV e outras infecções sexualmente transmissíveis foram afetadas na pandemia. Os serviços de saúde adotaram medidas de restrição nos atendimentos ambulatoriais, traduzida com diminuição do número de consultas, diminuição na rotina de testagem, mudanças nas atividades assistências e educativas nos Programas de Saúde, consultas de rotina canceladas e/ou reagendadas e redução do quantitativo 
de insumos e medicamentos, o que afetou significativamente a distribuição de medicamentos aos pessoas vivendo cadastrados nos serviços.

Pelos dados registrados na plataforma das redes de cuidados prestados em saúde observa-se que os serviços de saúde foram afetados e os gestores precisaram se reorganizar mediante as novas prioridades impostas pela pandemia, levando-se em consideração o risco-benefício em relação à segurança da população e dos profissionais de saúde.

Para melhor explanar os achados, exibiremos um quadro sinóptico (Quadro 1) elaborado após análise da produção científica disponível sobre a temática do impacto do isolamento social na pandemia de COVID-19 nos serviços de saúde no atendimento as pessoas que vivem com HIV.

Quadro 1. Síntese dos estudos sobre o impacto do isolamento social da pandemia da COVID-19 nos serviços de HIV.

\begin{tabular}{|c|c|c|c|c|c|}
\hline $\mathbf{N}^{\mathbf{o}}$ & TÍTULO & PERIÓDICO & OBJETIVO & RESULTADO & MÉTODO \\
\hline 1 & $\begin{array}{l}\text { Adapting to the } \\
\text { COVID-19 Pandemic: } \\
\text { Continuing HIV } \\
\text { Prevention Services } \\
\text { for Adolescents } \\
\text { Through } \\
\text { Telemonitoring, } \\
\text { Brazil }\end{array}$ & AIDS and Behavior & 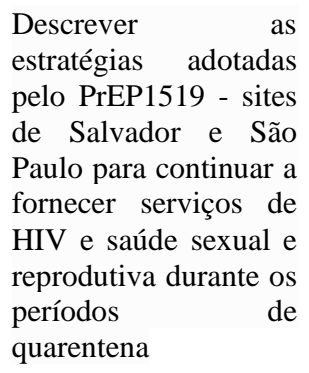 & $\begin{array}{l}\text { As plataformas de telessaúde } \\
\text { simplificaram e agilizaram os } \\
\text { procedimentos para o início da } \\
\text { PrEP e facilitou e possibilitou } \\
\text { a continuidade dos serviços da } \\
\text { PrEP durante a quarentena }\end{array}$ & $\begin{array}{lr}\text { Narrativa } & \text { com } \\
\text { análise } & \text { de } \\
\text { conteúdo } & \end{array}$ \\
\hline 2 & $\begin{array}{l}\text { COVID-19 Pandemic } \\
\text { Disrupts HIV } \\
\text { Continuum of Care } \\
\text { and Prevention: } \\
\text { Implications for } \\
\text { Research and Practice } \\
\text { Concerning } \\
\text { Community-Based } \\
\text { Organizations and } \\
\text { Frontline Providers }\end{array}$ & AIDS and Behavior & $\begin{array}{l}\text { Avaliar até que ponto } \\
\text { a pandemia de } \\
\text { COVID-19 } \\
\text { interrompeu } \\
\text { processo contínuo de } \\
\text { cuidado e prevenção } \\
\text { do HIV nas } \\
\text { organizações baseadas } \\
\text { na comunidade } \\
(\mathrm{CBOS})\end{array}$ & $\begin{array}{l}\text { A pandemia COVID-19 } \\
\text { apresenta desafios para } \\
\text { Community-Based } \\
\text { Organizations (CBOs) de } \\
\text { serviços de HIV, incluindo a } \\
\text { escassez de recursos, baixo } \\
\text { moral da equipe, interrupção } \\
\text { da prestação de serviços } \\
\text { centrada no paciente e déficits } \\
\text { orçamentários para CBOs que } \\
\text { dependem fortemente das } \\
\text { receitas do programa }\end{array}$ & $\begin{array}{lr}\text { Narrativa } & \text { com } \\
\text { análise } & \text { de } \\
\text { conteúdo } & \end{array}$ \\
\hline 3 & $\begin{array}{l}\text { HIV Care Experiences } \\
\text { During the COVID-19 } \\
\text { Pandemic: Mixed- } \\
\text { Methods Telephone } \\
\text { Interviews } \\
\text { Clinic-Enrolled HIV- } \\
\text { Infected Adults in } \\
\text { Uganda }\end{array}$ & $\begin{array}{l}\text { Nature Public Health } \\
\text { Emergency Collection }\end{array}$ & $\begin{array}{l}\text { Contribuir com as } \\
\text { evidências atualmente } \\
\text { limitadas, usando } \\
\text { entrevistas } \\
\text { telefone com questões } \\
\text { quantitativas } \\
\text { qualitativas para } \\
\text { examinar como os } \\
\text { clientes percebiam o } \\
\text { COVID-19 e seu } \\
\text { efeito no tratamento } \\
\text { do HIV e na adesão à } \\
\text { TARV }\end{array}$ & $\begin{array}{l}76 \% \text { dos clientes indicaram } \\
\text { que COVID-19 impactou } \\
\text { negativamente as viagens para } \\
\text { clínicas de HIV; } 54 \% \\
\text { perceberam que vir à clínica } \\
\text { aumentava o risco de adquirir } \\
\text { COVID-19; e } 14 \% \text { disseram } \\
\text { que COVID-19 teve um } \\
\text { impacto negativo na adesão ao } \\
\text { TARV. O feedback } \\
\text { qualitativo sugere que o medo } \\
\text { da infecção por COVID-19 } \\
\text { desencorajou a frequência à } \\
\text { clínica, enquanto os pedidos } \\
\text { de permanência em casa } \\
\text { ajudaram a rotinizar a adesão } \\
\text { ao TARV }\end{array}$ & $\begin{array}{lr}\text { Simulação } & \\
\text { realística } & \text { com } \\
\text { análise } & \text { de } \\
\text { conteúdo } & \end{array}$ \\
\hline
\end{tabular}




\begin{tabular}{|c|c|c|c|c|c|}
\hline 4 & $\begin{array}{lr}\text { Implications } & \text { of } \\
\text { COVID-19 for HIV } \\
\text { Research: r } \\
\text { sources, indicators } \\
\text { and } \\
\text { analyses }\end{array}$ & $\begin{array}{l}\text { Journal of the } \\
\text { International } \\
\text { AIDS Society }\end{array}$ & $\begin{array}{l}\text { Informar as diretrizes, } \\
\text { políticas e a prática de } \\
\text { prestação de serviços } \\
\text { de HIV }\end{array}$ & $\begin{array}{l}\text { O teste de HIV, o diagnóstico } \\
\text { e a vinculação aos cuidados } \\
\text { foram atrasados. As visitas } \\
\text { clínicas ambulatoriais foram } \\
\text { mudadas para encontros } \\
\text { remotos, e o atendimento não } \\
\text { urgente foi adiado. Atrasos no } \\
\text { início da TARV e recarga } \\
\text { ocorreram }\end{array}$ & $\begin{array}{l}\text { Narrativa } \\
\text { observacional } \\
\text { com análise de } \\
\text { conteúdo }\end{array}$ \\
\hline 5 & $\begin{array}{l}\text { PEPFAR's response } \\
\text { to the convergence of } \\
\text { the HIV and COVID- } \\
19 \text { pandemics in Sub- } \\
\text { Saharan Africa }\end{array}$ & $\begin{array}{l}\text { Journal of the } \\
\text { International } \\
\text { AIDS } \\
\text { Society }\end{array}$ & $\begin{array}{l}\text { Analisar a orientação } \\
\text { técnica COVID - } 19 \\
\text { do PEPFAR e } \\
\text { fornecer exemplos } \\
\text { específicos de países } \\
\text { de adaptações de } \\
\text { programas na África } \\
\text { Subsaaria }\end{array}$ & $\begin{array}{l}\text { As adaptações da prestação de } \\
\text { serviços de HIV resultantes de } \\
\text { interrupções do COVID-19 } \\
\text { requerem monitoramento e } \\
\text { avaliação cuidadosos. As } \\
\text { plataformas apoiadas pelo } \\
\text { PEPFAR estão mitigando os } \\
\text { impactos adversos do } \\
\text { COVID-19 nos serviços de } \\
\text { HIV, bem como nas próprias } \\
\text { pessoas vivendo com HIV }\end{array}$ & $\begin{array}{lr}\text { Narrativa } & \text { que } \\
\text { orienta } & \text { a } \\
\text { adaptação dos } & \text { serviços de HIV }\end{array}$ \\
\hline 6 & $\begin{array}{l}\text { Potential Influences of } \\
\text { the COVID-19 } \\
\text { Pandemic on Drug } \\
\text { Use and HIV Care } \\
\text { Among People Living } \\
\text { with HIV and } \\
\text { Substance r Use } \\
\text { Disorders: Experience } \\
\text { from a Pilot mHealth } \\
\text { Intervention }\end{array}$ & AIDS and Behavior & $\begin{array}{l}\text { Comparar o uso de } \\
\text { substâncias e os } \\
\text { cuidados com o HIV } \\
\text { antes e durante a } \\
\text { pandemia, usando } \\
\text { dados coletados } \\
\text { semanalmente por } \\
\text { meio de prevenção de } \\
\text { recaída de opióides e } \\
\text { intervenção de saúde } \\
\text { móvel de } \\
\text { gerenciamento de HIV }\end{array}$ & $\begin{array}{l}\text { Durante a pandemia, pessoas } \\
\text { vivendo com HIV e que } \\
\text { sofrem com transtorno de uso } \\
\text { de substâncias aumentaram o } \\
\text { uso de substâncias ilícitas e } \\
\text { diminuíram sua confiança } \\
\text { para permanecer sóbrios e } \\
\text { comparecer a reuniões de } \\
\text { recuperação. A proporção de } \\
\text { pessoas que perderam seus } \\
\text { medicamentos para o HIV } \\
\text { também aumentou e a } \\
\text { confiança para comparecer às } \\
\text { consultas de acompanhamento } \\
\text { de HIV diminuiu }\end{array}$ & $\begin{array}{l}\text { Prática clínica } \\
\text { com aplicação } \\
\text { de questionário }\end{array}$ \\
\hline 7 & $\begin{array}{lr}\text { Symptoms, } & \text { Stress, } \\
\text { and HIV-related care } \\
\text { among older people } \\
\text { living with } \text { HIV } \\
\text { during the COVID-19 } \\
\text { pandemic, Miami, } \\
\text { Florida }\end{array}$ & $\begin{array}{l}\text { Nature Public } \\
\text { Health Emergency } \\
\text { Collection }\end{array}$ & $\begin{array}{l}\text { Manter contato com } \\
\text { os participantes da } \\
\text { pesquisa e oferecer } \\
\text { uma opção para a } \\
\text { coleta de dados } \\
\text { adicionais. Também } \\
\text { ajudando-os a se } \\
\text { manterem conectados } \\
\text { e se colocar em } \\
\text { posição de ajudá-los } \\
\text { em caso de } \\
\text { emergência }\end{array}$ & $\begin{array}{l}\text { A maioria dos participantes } \\
\text { continuam recebendo } \\
\text { cuidados de HIV } \\
\text { pessoalmente, embora a } \\
\text { telessaúde tenha sido relatada } \\
\text { por alguns e possa ser uma } \\
\text { alternativa importante para os } \\
\text { cuidados pessoais no futuro }\end{array}$ & $\begin{array}{l}\text { Prática clínica } \\
\text { com aplicação } \\
\text { de questionário }\end{array}$ \\
\hline 8 & $\begin{array}{l}\text { The burden of anxiety } \\
\text { among people living } \\
\text { with HIV during the } \\
\text { COVID-19 pandemic } \\
\text { in Pune, India }\end{array}$ & BMC Public Health & $\begin{array}{l}\text { Compreender o fardo } \\
\text { da ansiedade e suas } \\
\text { fontes entre as pessoas } \\
\text { vivendo com HIV } \\
\text { indianas asiáticas } \\
\text { durante a pandemia } \\
\text { COVID }\end{array}$ & $\begin{array}{l}\text { No geral, a prevalência de } \\
\text { ansiedade generalizada foi de } \\
25 \% \text { ). As pessoas vivendo } \\
\text { com HIV com pontuação } \\
\text { GAD-7 } \geq 10 \text { tiveram menos } \\
\text { doses restantes de ART do } \\
\text { que aquelas com pontuações } \\
\text { GAD-7 mais baixas ( p = } \\
0,05) \text { A análise temática } \\
\text { indicou que as preocupações } \\
\text { eram relacionadas ou não à }\end{array}$ & $\begin{array}{l}\text { Prática clínica } \\
\text { com aplicação } \\
\text { de questionário }\end{array}$ \\
\hline
\end{tabular}




\begin{tabular}{|c|c|c|c|c|c|}
\hline & & & & $\begin{array}{lr}\text { saúde, e } & \text { afirmadas } \\
\text { temporalmente. } & \text { As } \\
\text { preocupações atuais muitas } \\
\text { vezes também foram } \\
\text { projetadas como preocupações } \\
\text { futuras }\end{array}$ & \\
\hline 9 & $\begin{array}{l}\text { The Impact of } \\
\text { COVID-19 on HIV } \\
\text { treatment } \\
\text { research: A call to } \\
\text { action }\end{array}$ & $\begin{array}{l}\text { International Journal } \\
\text { of Environmental } \\
\text { Research and Public } \\
\text { Health }\end{array}$ & $\begin{array}{lr}\text { (1) delinear } & \text { as } \\
\text { semelhanças } & \text { e } \\
\text { diferenças entre } & \text { o } \\
\text { COVID-19 e } & \text { as } \\
\text { pandemias de HIV; } \\
\text { (2) descrever o } \\
\text { impacto atual e futuro } \\
\text { do COVID-19 em } \\
\text { PWH; e (3) delinear } \\
\text { um apelo à ação para } \\
\text { cientistas r e } \\
\text { profissionais para } \\
\text { responder ao impacto } \\
\text { do COVID-19 na } \\
\text { prevenção ra e } \\
\text { tratamento do HIV }\end{array}$ & $\begin{array}{l}\text { Indivíduos com condições } \\
\text { crônicas de saúde, como o } \\
\text { HIV, podem desenvolver uma } \\
\text { resposta ao estresse mais forte } \\
\text { do que o resto da população. } \\
\text { É essencial reconhecer que } \\
\text { todas as pandemias têm } \\
\text { implicações biológicas, } \\
\text { psicológicas e sociais, das } \\
\text { quais os profissionais de } \\
\text { saúde desempenham um papel } \\
\text { crucial na preparação e na } \\
\text { resposta global }\end{array}$ & $\begin{array}{lr}\text { Narrativa } & \text { com } \\
\text { análise } & \text { de } \\
\text { conteúdo } & \end{array}$ \\
\hline
\end{tabular}

Fonte: Autoras (2020).

Ao realizar a leitura minuciosa dos nove artigos na busca de compreender o impacto do isolamento social na pandemia de COVID-19 no acesso ao tratamento e aos serviços de prevenção do HIV, podemos perceber que os serviços foram influenciados diretamente com as mudanças emergenciais adotadas para conter a COVID-19. Os artigos sobre a temática foram publicados em periódicos nacionais e internacionais, apresentando predominância artigos em idioma inglês e espanhol. Houve mudanças no atendimento, início, adesão e continuação da TARV. Existiu um aumento no atendimento remoto e flexibilização na distribuição de medicamentos. Deve-se dar ênfase às implicações psicológicas e sociais que a pandemia desenvolveu em respostas aos acontecimentos de mudanças, perdas de vidas e econômicas.

\section{Discussão}

Foi realizada uma análise qualitativa dos resultados evidenciados e encontramos três categorias a serem desmembradas, serviços de HIV e a pandemia de COVID-19, enfrentamento ao acesso dos serviços de HIV e saúde mental, estressores e sua influência no acesso aos serviços de HIV.

Pessoas vivendo com HIV que apresentam seu sistema imune reconstituído, não correm maior risco para infecção por Coronavírus, porém o isolamento social reverbera em barreiras e desafios para a continuidade do cuidado e testagem dos usuários. A interrupção do tratamento pode repercutir na resistência do vírus, então é imprescindível a continuação do tratamento. A redução do acesso à testagem de rotina traz desafios à primeira meta 90-90-90 do UNAIDS, de que todas as pessoas que vivem com HIV conheçam seu estado sorológico (Jiang et al., 2020).

Pessoas vivendo com HIV que deveriam ter iniciado a TARV no hospital podem ter encontrado barreiras que reverberam no impedimento ou atraso devido a grande demanda do Coronavírus, que resultou na realocação dos leitos, mudanças estruturais e de pessoal. Globalmente as autoridades de saúde pública estão focadas no controle de COVID-19, o que pode ser motivo para colocar os recursos para HIV em segundo plano. Algumas instituições internacionais, em colaboração com governos e parceiros da comunidade, estão trabalhando para sustentar a prestação de serviços de HIV (Jiang 
et al., 2020). Os serviços de saúde de diversas partes do mundo disponibilizam autoteste de HIV, porém mesmo com sua disponibilidade em algumas áreas, o acesso ainda enfrenta desafios, pois a demanda de serviços precisa considerar o ser humano como um ser biopsicossocial necessitando de um atendimento integral e individual, a dificuldade de acesso e a estigmatização da doença também deve ser considerada.

Para as pessoas que vivem com HIV a COVID-19 é uma doença grave, como na população em geral, porém pessoas idosas vivendo com HIV ou pessoas vivendo com HIV com problemas cardíacos e ou pulmonares, entre outras complicações podem apresentar maiores riscos e propensões a terem sintomas graves devido às suas vulnerabilidades. É emergencial que todas as pessoas que vivem com HIV procurem suas unidades e profissionais de saúde referência, para garantir que tenham estoques adequados de medicamentos essenciais e que suas prescrições sejam liberadas. Além de ser responsabilidade do sistema de saúde a integralidade no atendimento. Tendo em vista as limitações enfrentadas para a locomoção e atendimento na pandemia. Apesar da expansão do tratamento do HIV nos últimos anos, 15 milhões de pessoas vivendo com HIV não têm acesso à terapia antirretroviral, o que pode comprometer seu sistema imunológico (UNAIDS, 2020b). Para pessoas vivendo com HIV, abandonar o tratamento oferece maior propensão a risco de complicações do Coronavírus e maior chance de contaminação, uma vez que podem ter imunossupressão.

Segundo a OMS (2020) o autoteste é um processo no qual uma pessoa coleta sua própria amostra (fluido oral ou sangue) e, em seguida, realiza um teste e interpreta o resultado sozinho ou com alguém que confia. Refletindo em um importante auxílio na situação atual. No Brasil a atenção primária vivencia um novo desafio com o Coronavírus, afastando muitas pessoas desse atendimento, que é um importante aliado na prevenção e tratamento podendo evitar o adoecimento e diminuir o risco de transmissão, no SUS o acompanhamento e tratamento é garantido para todos.

\section{Serviços de HIV e a pandemia de COVID-19}

Os cuidados de prevenção recomendados para a redução da exposição ao COVID-19 são os mesmos para toda a população, a educação deve dar ênfase aos grupos populacionais que apresentam maior susceptibilidade. Pessoas com HIV devem continuar ativamente no atendimento e aconselhamento sobre o uso de preservativos com a mesma abordagem que tiveram para prevenir novas infecções por HIV e outras infecções sexualmente transmissíveis (ISTs), além de ter a sua disponibilidade gratuitamente para todos. Serviços de prevenção precisam se reorganizar para fornecer informações e educação em saúde a toda população. Há uma grande preocupação sobre a situação socioeconômica, a população migrante e a precariedade do sistema de saúde em algumas regiões que podem inviabilizar o cumprimento das medidas de prevenção na população mais vulnerável.

Pode-se esperar uma diminuição do diagnóstico de casos novos com o consequente aumento do diagnóstico tardio a médio e longo prazo, levando-se em consideração os riscos psicossociais e a interrupção dos serviços de saúde, tanto para controle quanto para diagnóstico precoce. É importante avaliar o risco potencial de diminuição da disponibilidade de RNA do HIV como teste de controle do tratamento. A prevenção da infecção pelo HIV é outro ponto a ser considerado e requer uma análise criteriosa, pois a saturação dos serviços de emergência podem levar a dificuldades no acesso à rota da profilaxia pósexposição (PEP) em casos de exposição ocupacional e não ocupacional, bem como à desaceleração da profilaxia pré-exposição (PREP) estratégia, que ainda está em fase de implementação em muitos países, especialmente na América Latina (Pousada -V, 2020).

A necessidade de avaliar até que ponto a pandemia de COVID-19 interrompeu o processo contínuo de cuidado e prevenção do HIV, ou seja, teste, profilaxia pré-exposição (PrEP) faz conexão com a proposta de um curso de ação para que possamos encerrar a epidemia de HIV nesta década. As interrupções causadas pelo COVID-19 nos permitem ver como a 
continuação dos cuidados com o HIV tem sido prejudicado rotineiramente por recursos organizacionais concretos e humanos insuficientes e por falhas em acompanhar e rastrear encaminhamentos de provedores para serviços de HIV. A busca por examinar a literatura atual e identificar não apenas lacunas, mas também as limitações de pesquisas se faz relevante para que possa ajudar os pesquisadores a abordarem as evidências limitadas sobre as práticas de encaminhamento e vinculação que podem auxiliar os clientes a acessar os serviços de HIV para os quais são encaminhados ("conclusão do encaminhamento") e, em última instância, acabar com a epidemia de HIV nesta década (Pinto \& Park, 2020).

O impacto em um grupo de 16 idosos participantes de um ensaio clínico, que vivem com HIV e em estado de imunossupressão avançada em Miami na Flórida é colossal. O medo da exposição potencial ao SARS-CoV-2, ajuste ao distanciamento social e problemas relacionados às finanças são os maiores estressores. Na pesquisa foi possível analisar os efeitos deletérios do distanciamento e a necessidade de intervenções que pensam além da doença transmissível mas também em alternativas que possam buscar o equilíbrio psicossocial e espiritual. Apesar de não serem relatados grandes impactos no tratamento e na distribuição de medicamentos de HIV, a pesquisa mencionou a transição da visita presencial para a telessaúde. O contato continuou semanalmente por meio de ligações telefônicas e observou a necessidade de conexão e atenção, na tentativa de diminuir medos, estressores e políticas de renda alternativas (Angel et al., 2020).

Embora não esteja claro se as pessoas que vivem com HIV têm risco aumentado de aquisição de SARS-CoV-2 ou progressão para doença grave, evidências emergentes da África do Sul sugerem que pode haver um aumento modesto na mortalidade associada ao vírus da imunodeficiência humana, independentemente do uso de TARV ou carga viral, mas especialmente na presença de outras comorbidades. Essa população está em alto risco de sofrer as consequências do impacto prejudicial do COVID-19 sobre os sistemas de saúde fracos e sobrecarregados. Ainda mais preocupantes, os instrumentos de laboratório, suprimentos e pessoal necessários para o monitoramento da carga viral, diagnóstico precoce do HIV e teste de tuberculose podem ser desviados para o teste de SARS-CoV-2. O resultado líquido será a incapacidade de atender às necessidades de prevenção, teste e tratamento para HIV. O medo da transmissão associada à saúde pode exacerbar a escassez de profissionais de saúde e desencorajar as pessoas a procurarem serviços essenciais de HIV nas instalações, muitos trabalhadores da saúde foram impactados pelo COVID-19 devido à quantidade insuficiente de equipamentos de proteção individual e à exposição. As estratégias de mitigação do COVID-19 estão limitando a importação e distribuição de produtos de saúde essenciais necessários para serviços essenciais de HIV. Além disso, surgiram preocupações de que os planos do COVID19 desencaminharam recursos dos programas de HIV, Tuberculose e Malária (Golin et al., 2020).

Embora muitos serviços de saúde estejam oferecendo serviços de telessaúde, a transição para a telessaúde tem sido lenta em alguns lugares, e a aceitação dos serviços de telessaúde é variável. Alguns usuários podem não ter os recursos necessários para aproveitar plenamente as oportunidades de telessaúde (por exemplo, acesso a dispositivos ou serviço de Internet adequado). À medida que as unidades de saúde reabrem e retomam as atividades normais, os usuários dos serviços enfrentam o risco de exposição ao COVID-19 ao comparecer às consultas médicas. Dadas as altas taxas de problemas de saúde mental (por exemplo, ansiedade e depressão) que existem entre esta população, eles se tornam vulneráveis aos efeitos do isolamento resultantes da restrição de mobilidade associada à pandemia. Além disso, na medida em que o COVID-19 está afetando as comunidades marginalizadas de maneiras únicas, também está afetando essa população de maneiras únicas, visto que o HIV afeta desproporcionalmente as pessoas de grupos marginalizados. O impacto desproporcional da pandemia sobre as pessoas de etnias marginalizadas, imigrantes e aqueles que são economicamente desfavorecidos está tornando aparentes as disparidades de saúde existentes que também existem dentro da epidemia de HIV (Chenneville et al., 2020). 


\section{Medidas de acesso aos serviços de HIV}

De acordo com Golin et al (2020) as plataformas apoiadas pelo President's Emergency Plan for AIDS Relief (PEPFAR) estão mitigando os impactos adversos do COVID-19 nos serviços de HIV. Em antecipação o PEPFAR estabeleceu uma orientação para adaptar rapidamente os programas de HIV, mantendo os serviços essenciais de HIV, protegendo os destinatários dos cuidados e os funcionários da linha de frente da pandemia. Congruente com a recente defesa da International AIDS Society há uma ênfase especial na continuidade do tratamento dos clientes por meio do uso expandido da dispensação descentralizada e por vários meses de antirretrovirais. A prestação ininterrupta de serviços essenciais de prevenção, incluindo profilaxia infantil de HIV, profilaxia pré-exposição ao HIV, cuidados pré e pós-natais, planejamento familiar e imunizações infantis também é priorizada, com orientações para descentralização dos serviços o máximo possível. Recomendações para monitoramento clínico virtual e outros serviços essenciais, incluindo suporte de laboratório e gerenciamento de commodities, foram incluídos.

O PEPFAR em resposta a demanda de apoio psicossocial necessária orienta e apoio o fornecimento de atendimento por meio de plataformas virtuais, como ligações e plataformas de mídia social (por exemplo, Facebook e WhatsApp), podendo substituir os serviços presenciais, ao mesmo tempo que reduzem o risco de transmissão de SARS-CoV-2 para clientes e funcionários. As estratégias de controle do COVID-19 resultaram em aumentos relatados na violência doméstica e podem aumentar os riscos de proteção infantil. Órfãos, crianças vulneráveis, meninas adolescentes e mulheres jovens foram priorizados para receber triagem de risco virtual e vinculação a serviços essenciais, incluindo encaminhamento se estiver sob risco ou sofrendo abuso, negligência ou violência. Orientações específicas foram fornecidas sobre como fornecer apoio virtual aos beneficiários do programa Determinado, Resiliente, Empoderado, Orientado e Seguro (Golin et al., 2020).

A resposta global ao HIV resume a convergência de políticas e saúde pública. No início, foi amplamente caracterizada por esforços políticos globais. Os esforços de base entre os mais afetados pelo HIV serviriam como a resposta global inicial à pandemia. Muitos governos foram criticados por sua resposta lenta e moralista. Foram ativistas e organizações sem fins lucrativos não governamentais que se organizaram para defender uma resposta governamental imediata e fornecer educação e apoio às comunidades locais afetadas. No entanto, a resposta do governo desempenhou um papel complicado na história do HIV. Os governos, ou melhor, suas políticas, têm desempenhado um papel significativo na proibição do progresso, incluindo leis que criminalizam o comportamento de pessoas vivendo com HIV. O que recaí na restrição de serviços para populaçõeschave; acesso limitado a TARV; falha em fornecer educação sexual abrangente em ambientes escolares; e suporte insuficiente para programas de redução de danos. Além disso, o apoio financeiro global para o HIV diminuiu continuamente nos últimos anos (Chenneville et at, 2020).

O PrEP1519 é o primeiro estudo de coorte de demonstração na América Latina que explora a aceitabilidade, o Brasil tenha lançado a PrEP em seu sistema nacional de saúde em 2018. A fim de minimizar os efeitos da quarentena no início da PrEP, na continuação da PrEP e no acesso a outros cuidados de saúde sexual e necessidades de prevenção, foram alterados os processos de pesquisa e serviços de atendimento. No estudo PrEP1519 realizado em São Paulo e Salvador a adaptação aos serviços oferecidos a adolescentes se deu através dos serviços de telessaúde combinado como plataformas de prestação de cuidados padrão, como recrutamento de participantes digitais e suporte de pares e navegação por mensagens de texto em smartphones e mídia social online (Instagram e Facebook), procedimentos simplificados de iniciação e retenção de PrEP. As estratégias de navegação online foram mais intensificadas à medida que os adolescentes estavam ansiosos para conversar e fazer perguntas sobre como persistir com o uso da PrEP em quarentena. Para início da PreP uma consulta presencial é agendada, seguindo a rotina pré COVID-19, porém com tempo reduzido em até 50\%, devido ao atendimento online. Para a continuação da PrEP, aqueles que a equipe de saúde identifica como elegíveis, uma consulta de telessaúde é agendada e o 
medicamento PrEP e auto-teste de HIV são entregues em seus endereços. Para aqueles que não são elegíveis ou o participante não deseja o atendimento online, é agendada uma consulta presencial. Atendimentos de Saúde mental também estão disponíveis nos mecanismos de telessaúde dos participantes (Dourado et al., 2020).

$\mathrm{O}$ estudo de Uganda para contribuir com evidências realizou entrevistas por telefone com questões quantitativas e qualitativas para examinar como os clientes percebiam o COVID-19 e seu efeito no tratamento do HIV e na adesão à TARV. O feedback qualitativo sugere que o medo da infecção por COVID-19 desencorajou a frequência à clínica, enquanto os pedidos de permanência em casa ajudaram a rotinizar a adesão à TARV e empregar novas abordagens baseadas na comunidade para cuidados de HIV (Linnemayr et al., 2020).

Neste cenário é de extrema urgência a busca do conhecimento das barreiras relacionadas ao tratamento adequado do adoecimento físico e psicossocial entre outras demandas do contexto social das pessoas vivendo com HIV, sendo uma importante ferramenta no rastreio de possibilidades para oferecer uma assistência de mais qualidade a esta população (Souza et al., 2020).

\section{Saúde Mental, estressores e sua influência no acesso aos serviços de HIV}

Transtornos psiquiátricos como ansiedade, depressão, estresse pós-traumático e uso de substâncias são comuns entre pessoas vivendo com HIV, sendo considerados como uma causa, quanto uma consequência do HIV e muito pelo estigma e preconceitos que essas pessoas começam a vivenciar. Tal associação evidencia-se no fato de a prevalência desses transtornos na população vivendo com HIV ser maior do que na população em geral (Camargo et al., 2020). Da mesma forma, o medo de pandemia em geral pode piorar os transtornos mentais existentes e também pode resultar em novos diagnósticos. Para COVID19 especificamente, o isolamento social tende a contribuir para os transtornos mentais (Chenneville et al., 2020).

A ansiedade está associada à não adesão à terapia antirretroviral (TARV), corroborando no afastamento os cuidados. Do ponto de vista da saúde pública, é importante compreender a relação entre a saúde mental entre pessoas vivendo com HIV, os resultados do tratamento do HIV e a dinâmica de transmissão do HIV. A depressão está associada ao fracasso do tratamento, contagens de CD4 mais baixas e comportamento sexual. A importância da geração de dados de saúde mental entre pessoas vivendo com HIV no cenário da pandemia pode ser inferida, visto que a piora da saúde mental foi relatada na população em geral. Na análise temática foram identificados os seguintes quatro temas: a) preocupações relacionadas com o presente imediato; b) preocupações relacionadas ao futuro iminente; c) falta de apoio social e financeiro; d) indiferença às circunstâncias secundárias ao COVID-19 (Marbaniang et al., 2020). Uma saúde mental equilibrada diminui os riscos de pouca adesão e abandono ao tratamento. Por isso, fornecer atendimento psicossocial precisa ser garantido nos serviços de saúde junto a medidas e ações contínuas pelo fim do estigmatiza e preconceito a população-chave.

\section{Conclusão}

É necessário manter um acompanhamento próximo no atendimento a essa população com estratégias de informação e educação continuada, enfatizando a adesão à TARV e medidas de prevenção do contágio por meio do acompanhamento e apoio ao programa multidisciplinar com tecnologias de comunicação otimizadas, para atingir os objetivos de identificação precoce dos sintomas da COVID-19, seu diagnóstico e tratamento precoces e a prevenção de desfechos críticos ou fatais pela coinfecção com este novo Coronavírus. É crucial expandir o conhecimento sobre os efeitos da COVID-19 na infecção pelo HIV nos níveis local, regional e global. 
É necessária a busca por alternativas para que os atendimentos presenciais nas unidades de saúde sejam separados ou tenham horários específicos. Se não se estabelecerem fluxos específicos para facilitar o acesso, os principais riscos serão: atrasar o diagnóstico de pessoas que desconhecem seu estado sorológico e também de novos casos de HIV e outras IST's. Essas experiências oferecem lições importantes para programas e políticas de prevenção combinada do HIV e outras IST's em questão. Os programas devem promover o desenvolvimento de alternativas para assegurar a distribuição de autotestes de HIV utilizando plataformas online, correios, pelos pares e comunidades, em locais onde as populações-chave se localizam.

O desafio é manter a pauta e avançar nas respostas à epidemia do HIV em tempos de COVID-19, em um momento tão crítico, o avanço da cobertura universal e universalidade e equidade no cuidado deve se fazer presente. Em resposta a esses desafios, a OMS, a UNAIDS, Rede Global de Pessoas Vivendo com HIV, ONGs e coletivos estão trabalhando juntos para garantir a prestação contínua de serviços de prevenção, teste e tratamento do HIV. Constata-se que além de uma reorganização estrutural foi necessária uma flexibilização dos serviços que atendem pessoas vivendo com HIV, em busca de erradicar interrupções dos atendimentos e violações dos direitos humanos contra populações vulneráveis e marginalizadas. Neste sentido, foi possível observar relatos das pessoas vivendo sobre como as estratégias para o enfrentamento do Coronavírus, influenciaram na continuidade e adesão ao tratamento antirretroviral, e nos serviços de prevenção ao HIV.

Para futuros estudos, espera-se o desenvolvimento de pesquisas que ofereçam subsídios para o fortalecimento do conhecimento científico, referente ao impacto gerado na resposta aos serviços de saúde que visem a atenção aos cuidados envolvendo prevenção, diagnóstico, controle e tratamento de HIV e outras IST's em tempos de pandemia e isolamento social. Espera-se contribuir para amplificação e realização de novas pesquisas que ofereçam maior reflexão sobre os impactos da pandemia na prestação de serviços no SUS, destacando os resultados mais concisos da repercussão das mudanças na prática assistencial nos diferentes programas de saúde.

\section{Referências}

Algarin, A. B., Varas-Rodríguez, E., Valdivia, C., Fennie, K. P., Larkey, L., Hu, N., \& Ibañez, G. E. (2020). Symptoms, Stress, and HIV-Related Care Among Older People Living with HIV During the COVID-19 Pandemic, Miami, Florida. AIDS and behavior, 24(8), 2236-2238. https://doi.org/10.1007/s10461-02002869-3.

Camargo, L. A., Capitão, C. G., \& Filipe, E. M. V. (2014). Saúde mental, suporte familiar e adesão ao tratamento: associações no contexto HIV/Aids. PsicoUSF, 19(2), 221-232. https://doi.org/10.1590/1413-82712014019002013.

Chenneville, T., Gabbidon, K., Hanson, P., \& Holyfield, C. (2020). The Impact of COVID-19 on HIV Treatment and Research: A Call to Action. International journal of environmental research and public health, 17(12), 4548. https://doi.org/10.3390/ijerph17124548.

Dias, J. A. A., Dias, M. F. S. L., Oliveira, M. O., Freitas, L. M. A. F., Santos, N. C. N., \& Freitas, C. A. F. (2020). Reflexões sobre distanciamento, isolamento social e quarentena como medidas preventivas da COVID-19. Revista de Enfermagem do Centro Oeste Mineiro, 2020;10:e3795 https://doi.org/10.19175/recom.v10i0.3795.

Dourado, I., Magno, L., Soares, F., Massa, P., Nunn, A., Dalal, S., Grangeiro, A., \& Brazilian PrEP1519 Study Group (2020). Adapting to the COVID-19 Pandemic: Continuing HIV Prevention Services for Adolescents Through Telemonitoring, Brazil. AIDS and behavior, 24(7), 1994-1999 https://doi.org/10.1007/s10461-020-02927-w.

FIO CRUZ. (2020). Covid-19 impacta tratamento de pessoas vivendo com HIV e HTLV. https://portal.fiocruz.br/noticia/covid-19-impacta-tratamento-depessoas vivendo-com-hiv-e-htlv.

Golin, R., Godfrey, C., Firth, J., Lee, L., Minior, T., Phelps, B. R., Raizes, E. G., Ake, J. A., \& Siberry, G. K. (2020). PEPFAR's response to the convergence of the HIV and COVID-19 pandemics in Sub-Saharan Africa. Journal of the International AIDS Society, 23(8), e25587. https://doi.org/10.1002/jia2.25587.

Hochstatter, K. R., Akhtar, W. Z., Dietz, S., Pe-Romashko, K., Gustafson, D. H., Shah, D. V., Krechel, S., Liebert, C., Miller, R., El-Bassel, N., \& Westergaard, R. P. (2020). Potential Influences of the COVID-19 Pandemic on Drug Use and HIV Care Among People Living with HIV and Substance Use Disorders: Experience from a Pilot mHealth Intervention. AIDS and behavior, 1-6. https://doi.org/10.1007/s10461-020-02976-1.

Jiang, H., Zhou, Y., \& Tang, W. (2020). Maintaining HIV care during the COVID-19 pandemic. The lancet. HIV, 7(5), e308-e309.

https://doi.org/10.1016/S2352-3018(20)30105-3. 
Research, Society and Development, v. 10, n. 1, e28110111692, 2021

(CC BY 4.0) | ISSN 2525-3409 | DOI: http://dx.doi.org/10.33448/rsd-v10i1.11692

Linnemayr, S., Jennings Mayo-Wilson, L., Saya, U., Wagner, Z., MacCarthy, S., Walukaga, S., Nakubulwa, S., \& Karamagi, Y. (2020). HIV Care Experiences During the COVID-19 Pandemic: Mixed-Methods Telephone Interviews with Clinic-Enrolled HIV-Infected Adults in Uganda. AIDS and behavior, 1-12. https://doi.org/10.1007/s10461-020-03032-8.

Ministério da Saúde. Entenda a diferença entre Coronavírus, Covid-19 e Novo Coronavírus. https://www.gov.br/pt-br/noticias/saude-e-vigilanciasanitaria/2020/03/entenda-a-diferenca-entre-coronavirus-covid- -19-e-novo-coronavirus.

Ministério da Saúde. Entenda a diferença entre Coronavírus, Covid-19 e Novo Coronavírus. https://www.gov.br/pt-br/noticias/saude-e-vigilanciasanitaria/2020/03/entenda-a-diferenca-entre-coronavirus-covid- -19-e-novo-coronavirus.

Ministério da Saúde. O que é HIV? http://www.aids.gov.br/pt-br/publico-geral/o-que-e-hiv.

Ministério da Saúde. Painel de casos de doença pelo coronavírus 2019 (COVID-19) no Brasil pelo Ministério da Saúde. https://covid.saude.gov.br/.

Ministério da Saúde. Prevenção combinada. http://www.aids.gov.br/pt-br/publico-geral/previna-se.

Ministério da Saúde. Tratamento para o HIV. http://www.aids.gov.br/pt-br/publico-geral/o-que-e-hiv/tratamento-para-o-hiv.

Marbaniang, I., Sangle, S., Nimkar, S., Zarekar, K., Salvi, S., Chavan, A., Gupta, A., Suryavanshi, N., Mave, V. (2020). The burden of anxiety among people living with HIV during the COVID-19 pandemic in Pune, India. BMC Public Health 20, 1598. https://doi.org/10.1186/s12889-020-09656-8.

Moher, D., Liberati, A., Tetzlaff, J., Altman D. G., \& The PRISMA Group. (2009) Preferred Reporting Items for Systematic Reviews and Meta-Analyses: The PRISMA Statement. PLOS Medicine 6(7): e1000097. https://doi.org/10.1371/journal.pmed.1000097.

Moura, Maria Lucia Costa de. (2020). Coronavírus e COVID-19. Revista Saúde Coletiva, 53, 10.

Organização Pan Americanas da Saúde. Brasil. (2020). A Organização Mundial de Saúde afirma que COVID-19 é agora caracterizada como pandemia. https://www.paho.org/bra/index.php?option=com_content\&view=article\&id=6120:oms-afirma-que-covid-19-e-agora-caracterizada-comopandemia\&Itemid $=812$

Pereira, A. S., Shitsuka, D. M., Parreira, F. J., \& Shitsuka, R. (2018). Metodologia da pesquisa científica: UFSM, NTE.

Peters, M. D. J., Godfrey, C. M., McInerney, P., Soares, C. B., Khalil, H., \& Parker, D. (2015). The Joanna Briggs Institute reviewers' manual 2015: methodology for JBI scoping reviews. Australia, The Joanna Briggs Institute.

Pinto, R. M., \& Park, S. (2020). COVID-19 Pandemic Disrupts HIV Continuum of Care and Prevention: Implications for Research and Practice Concerning Community-Based Organizations and Frontline Providers. AIDS and behavior, 24(9), 2486-2489. https://doi.org/10.1007/s10461-020-02893-3.

Rebeiro, P. F., Duda, S. N., Wools-Kaloustian, K. K., Nash, D., Althoff, K. N., \& International epidemiology Databases to Evaluate AIDS (IeDEA). (2020). Implications of COVID-19 for HIV Research: data sources, indicators and longitudinal analyses. Journal of the International AIDS Society, 23 (10), e25627. https://doi.org/10.1002/jia2.25627.

Santos, C. M. D. C., Pimenta, C. A. D. M., \& Nobre, M. R. C. (2007). A estratégia PICO para a construção da pergunta de pesquisa e busca de evidências. Revista Latino-Americana de Enfermagem, 15(3)

Souza, M. T., Silva, M. D., \& Carvalho, R. (2010). Integrative review: what is it? How to do it?. Einstein (São Paulo), 8(1), $102-106$. https://doi.org/10.1590/s1679-45082010rw1134.

Souza, T. de C., Monteiro, D. da R., Duarte, A. dos S., Trevisan, B. F., Tanaka, R. Y., \& Oliveira, T. da S. (2020). The impact of the HIV virus on the quality of life of the elderly: an integrative review. Research, Society and Development, 9(11), e90891110566. https://doi.org/10.33448/rsd-v9i11.10566.

UNAIDS. (2020a). Informações básicas. https://unaids.org.br/informacoes-basicas/.

UNAIDS (2020b). O que as pessoas que vivem com HIV precisam saber sobre HIV e COVID-19. https://unaids.org.br/2020/04/o-que-as-pessoas-que-vivemcom-hiv-precisam-saber-sobre-hiv-e-covid-19/. 